\title{
Magnetic Doppler imaging of the roAp star HD 24712 ${ }^{\star}$
}

\author{
T. Lüftinger ${ }^{1}$, O. Kochukhov ${ }^{2}$, T. Ryabchikova ${ }^{1,3}$, N. Piskunov ${ }^{2}$, W. W. Weiss ${ }^{1}$, and I. Ilyin ${ }^{4}$ \\ 1 Institut für Astronomie, Universität Wien, Türkenschanzstrasse 17, 1180 Wien, Austria \\ e-mail: lueftinger@astro.univie.ac.at \\ 2 Department of Physics and Astronomy, Uppsala University Box 515, 75120 Uppsala, Sweden \\ 3 Institute of Astronomy, Russian Academy of Sciences, Pyatnitskaya 48, 119017 Moscow, Russia \\ 4 Astrophysikalisches Institut Potsdam, An der Sternwarte 16, 14482 Potsdam, Germany \\ Received 18 December 2008 / Accepted 15 October 2009
}

\section{ABSTRACT}

\begin{abstract}
Aims. We present the first magnetic Doppler images of a rapidly oscillating Ap (roAp) star.
Methods. We deduce information about magnetic field geometry and abundance distributions of a number of chemical elements on the surface of the hitherto best studied roAp star, HD 24712, using the magnetic Doppler imaging (MDI) code, INVERS10, which allows us to reconstruct simultaneously and consistently the magnetic field geometry and elemental abundance distributions on a stellar surface. For this purpose we analyse time series spectra obtained in Stokes $I$ and $V$ parameters with the SOFIN polarimeter at the Nordic Optical Telescope and recover surface abundance structures of sixteen different chemical elements, respectively ions, including $\mathrm{Mg}$, Ca, Sc, Ti, Cr, Fe, Co, Ni, Y, La, Ce, Pr, Nd, Gd, Tb, and Dy. For the rare earth elements (REE) Pr and Nd separate maps were obtained using lines of the first and the second ionization stage.

Results. We find and confirm a clear dipolar structure of the surface magnetic field and an unexpected correlation of elemental abundance with respect to this field: one group of elements accumulates solely where the positive magnetic pole is visible, whereas the other group avoids this region and is enhanced where the magnetic equatorial region dominates the visible stellar surface. We also observe relative shifts of abundance enhancement- or depletion regions between the various elements exhibiting otherwise similar behaviour.
\end{abstract}

Key words. stars: imaging - stars: atmospheres - stars: abundances - stars: chemically peculiar - stars: magnetic field stars: individual: HD 24712

\section{Introduction}

Many physical processes important for stellar evolution are crucially influenced by stellar magnetic fields. Theoretical and observational frameworks tell us for instance that they influence microscopic diffusion of chemical elements within stellar atmospheres, and they may also redistribute angular momentum or give rise to enhanced hydrodynamical instabilities and thus modify mixing properties in stellar interiors.

Ap stars, representing about 5-10\% of the upper main sequence stars, exhibit magnetic fields that appear to be highly ordered, very stable and often very strong. Many Ap stars also show strong spectral line profile variations synchronized to stellar rotation. This is attributed to oblique magnetic and rotation axes and to the presence of a non-uniform distribution of chemical elements on their surface within the framework of the oblique rotator model (introduced by Stibbs 1950). With a few exceptions such inhomogeneities exist only in the atmospheres of A stars with magnetic fields, demonstrating that these fields play a crucial role in their formation and evolution.

The spectra of Ap stars also exhibit a remarkable variety of sometimes unidentified spectral line features. Ryabchikova et al. (2004) for example find overabundances of some $\mathrm{Fe}$ peak elements, which may reach a few dex for $\mathrm{Cr}$ and even higher for the REE, especially for their second ionization

^ Based on observations made with the Nordic Optical Telescope, operated on the island of La Palma jointly by Denmark, Finland, Iceland, Norway, and Sweden, in the Spanish Observatorio del Roque de los Muchachos of the Instituto de Astrofisica de Canarias. stage, while other chemical elements are found to be underabundant compared to the solar value. An important subgroup of the Ap stars, the rapidly oscillating Ap stars, in addition exhibits high-overtone, low-degree, non-radial $p$-mode pulsations with periods of 6-21 min (Kurtz \& Martinez 2000). Their observed pulsational amplitudes are modulated with the magnetic field variations, exhibiting maximum amplitude at the phases of highest magnetic field strength (Kurtz 1982). This indicates a close connection between the magnetic field and the excitation mechanism of roAp pulsations.

The chemical peculiarities mentioned are attributed to the selective diffusion of ions under the competitive action of radiative acceleration and gravitational settling (Michaud 1970) possibly in combination with a weak, magnetically driven wind (see, e.g., Babel 1992).

We focus our research on roAp stars since these objects exhibit a number of new, largely unexplored phenomena related to their pulsational variability while sharing all the properties of other Ap stars. The roAp stars are the only main-sequence stars in which high-overtone p-mode pulsations are easily detected and pulsation modes can be identified. Furthermore, roAp stars represent the only group of pulsating stars for which magnetic and pulsation characteristics can well be constricted observationally. Hence, peculiar pulsating stars are key objects in the investigation of the atmospheric and internal structure of the middle main-sequence stars.

Very little is known as yet about the origin and structure of magnetic fields and their connection and interaction with surface abundance patches and pulsation. With the development of 
high-resolution spectropolarimeters it has become possible to extract the full amount of information about magnetic field and abundance distributions using Stokes parameter observations and applying magnetic Doppler imaging (MDI).

We have developed and tested a code for MDI (Piskunov \& Kochukhov 2002; Kochukhov \& Piskunov 2002) that allows us to reconstruct simultaneously and consistently the magnetic field vector and abundance distribution of various elements on a stellar surface by taking into account all relevant physics of polarized line formation.

In deriving the surface abundances of numerous light-, ironpeak- and rare earth elements by applying MDI, we get more insight into the relation between the magnetic fields, vertical and horizontal abundance characteristics and pulsation, and thus into the atmospheric structure of Ap and roAp stars. At present surface abundance structures have been derived for only one other roAp star, HD 83368 (Kochukhov et al. 2004b).

Our paper is composed in the following way: Sect. 2 is devoted to the description of the target star, HD 24712, on which we comment in Sect. 3 regarding our spectropolarimetric observations and the data reduction. Details about MDI, the magnetic field geometry of HD 24712, and the surface abundance structures of individual elements can be found in Sect. 4 while Sect. 5 is devoted to the discussion of our results.

\section{HD 24712}

HD 24712 (HD1217, DO Eri) is one of the best-studied roAp stars with light (Wolff \& Morrison 1973), spectrum and magnetic variations that was discovered to pulsate by Kurtz (1982). Matthews et al. (1988) found radial velocity variations with an amplitude of $0.4 \pm 0.05 \mathrm{~km} \mathrm{~s}^{-1}$ and the main photometric period of $6.14 \mathrm{~min}$.

During an observing campaign with the Whole Earth Telescope (WET), Kurtz et al. (2002) detected a "missing" mode. The detailed investigations by Cunha et al. (2003, 2006) suggest in this context, that the $p$-mode pulsations are strongly affected by the global stellar magnetic field.

A study of the surface abundance inhomogeneity in HD 24712 based on the observed line profile variations was performed by Ryabchikova et al. (2000). Surface element distribution was schematically approximated as a set of circular spots whose position, radii and the abundances inside and outside the spots have been obtained with an iterative procedure. Ryabchikova et al. (2000) found that Pr II, III, Nd II, III, and Co are concentrated in one large spot near the magnetic pole, while $\mathrm{Fe}$ is concentrated rather in 4 spots around the magnetic equator. They also found that centers of $\mathrm{Nd}, \mathrm{Pr}$ and Co spots do not coincide with the pole of the magnetic field geometry determined by Bagnulo et al. (1995), but are shifted by $\approx 10^{\circ}$ towards the magnetic equator. According to their analysis Pr-Nd and Co are overabundant by $\approx+1$ dex in the spot relative to the outside surface area and one needs $\approx 2$ dex abundance differences between $\mathrm{Pr}$ and $\mathrm{Nd}$ in the first and the second ionization stages. The maximum surface abundance gradient for Fe was 0.6 dex.

We performed a detailed spectroscopic pulsation study of radial velocity variations in high resolution and high time-resolved data (obtained with SOFIN at NOT and with UVES at the ESO VLT). Simultaneously we performed high precision photometric observations from space with the MOST (Microvariability and Oscillation of Stars) satellite (Walker et al. 2003). During this process, we uncovered a detailed pulsation picture of the star (Ryabchikova et al. 2007). We found maximum radial velocity amplitudes for rare earth lines and in addition phase shifts
Table 1. Basic physical parameters of HD 24712.

\begin{tabular}{ll}
\hline \hline$T_{\text {eff }}$ & $7250 \pm 150 \mathrm{~K}$ \\
$\log g$ & $4.2 \pm 0.1$ \\
$M$ & $1.63 M_{\odot}$ \\
$L / L_{\odot}$ & $0.91 \pm 0.04$ \\
$P_{\text {rot }}(\mathrm{d})$ & $12.45877 \pm(16)$ \\
$P_{\text {puls }}$ & $\simeq 6.15 \mathrm{~min}$ \\
$v_{\mathrm{e}} \sin i$ & $5.6 \mathrm{~km} \mathrm{~s}^{-1}$ \\
\hline
\end{tabular}

progressively increasing from one element to another and within different groups of lines of the same element. We propose that this pulsational behaviour is caused by the fact that these elements are concentrated in a thin layer in the stellar atmosphere where pulsation reaches its maximum amplitude.

These observations and a possible correlation to theoretical investigations made HD 24712 an ideal candidate for spectroscopic and spectropolarimetric observations with ground based equipment.

The basic physical parameters of HD 24712 are listed in Table 1. $T_{\text {eff }}$ and $\log g$ were applied as derived by Ryabchikova et al. (1997). The atomic data used in our study was extracted from the Vienna Atomic Line Database (VALD, Piskunov et al. 1995; Ryabchikova et al. 1999; Kupka et al. 1999).

\section{Observations and data reduction}

Spectropolarimetric observations of HD 24712 were carried out in 2003, between Oct. 29 and Nov. 18, using the high-resolution échelle spectrograph SOFIN attached to the Cassegrain focus of the $2.56 \mathrm{~m}$ Nordic Optical Telescope (NOT), La Palma, Spain. The spectrograph is equipped with three different cameras, and to obtain observations in the spectropolarimetric mode, the second camera was used. For MDI we used 49 different spectral lines from seven spectral orders, each covering about $40 \AA$ to $50 \AA$ between 4000 and $6000 \AA$.

The polarized spectra were obtained with a Stokesmeter, consisting of a calcite plate used as a beam splitter and an achromatic rotating quarter-wave plate. The position of the quarterwave plate is controlled by a stepping motor. Usually a sequence of four subexposures is processed in order to obtain accurate circular polarization measurements, where each of the beams is exposed twice, with the quarter-wave plate rotated by $90^{\circ}$ after the first and before the last exposure. This way it is possible to reduce instrumental effects to a minimum, because in the images taken with the quarter-wave plate rotated by $90^{\circ}$, possible instrumental signatures change sign and cancel each other out during the averaging of the two exposures.

For data reduction, the $4 \mathrm{~A}$ software package written in $\mathrm{C}$ (Ilyin 2000) was used. All standard procedures like bias subtraction, flat field correction, subtraction of the scattered light, weighted extraction of the orders, and bad pixel (cosmic ray) corrections were included. ThAr exposures obtained before and after each observing night were used to perform wavelength calibration and to test for possible spurious instrumental polarization, caused for instance by bending of the spectrograph, which is directly mounted on the telescope, different positions of the star on the slit or temporal variations of the seeing. $S / N$ ratios for the observed spectra typically reached 500-600 per pixel at $\lambda=5000 \AA$, except for one spectrum which has $S / N \approx 300$. Reduced spectra have a resolving power of $\approx 40000$.

In total we obtained observations at 13 rotational phases. Each pair of the Stokes $I$ and $V$ spectra was calculated from a 
Table 2. Journal of spectropolarimetric observations of HD 24712.

\begin{tabular}{ccc}
\hline \hline $\begin{array}{c}\text { HJD } \\
(2450000+)\end{array}$ & Rotational phases & $S / N$ \\
\hline 2941.6516 & 0.4396 & 290 \\
2943.6341 & 0.6001 & 550 \\
2945.5758 & 0.7562 & 520 \\
2946.5973 & 0.8380 & 510 \\
2947.6067 & 0.9171 & 570 \\
2948.6514 & 0.0026 & 660 \\
2952.6267 & 0.3207 & 560 \\
2953.6572 & 0.4040 & 520 \\
2954.6316 & 0.4824 & 550 \\
2955.6350 & 0.5620 & 570 \\
2956.5954 & 0.6390 & 620 \\
2957.5958 & 0.7204 & 560 \\
2961.6160 & 0.0414 & 500 \\
\hline
\end{tabular}

sequence of polarimetric exposures obtained over $35 \mathrm{~min}$. This is sufficiently long to average out variations due to the $6.14 \mathrm{~min}$ pulsation period of HD 24712.

Rotation phases of HD 24712 (see Table 2) were calculated according to the ephemeris and rotation period derived by Ryabchikova et al. (2005):

$\operatorname{HJD}\left(\left\langle B_{\mathrm{Z}}\right\rangle_{\max }\right)=2453235.18(40)+12.45877(16) \mathrm{d}$.

\section{Magnetic Doppler Imaging (MDI)}

Doppler imaging (DI) has become a very successful tool to gain information on inhomogeneities on the surface of a rotating star caused by changing abundance and/or magnetic field structure. The first method for reconstruction of stellar magnetic field geometries from Stokes $V$ and $I$ spectropolarimetry was developed by Piskunov (1985) and applied to Ap stars by Glagolevskii et al. (1985). A similar technique has been very successfully applied to mapping magnetic field topologies in late-type active stars by Donati \& Collier Cameron (1997), Hussain et al. (2001) and Donati et al. (2006).

By inverting time series of spectropolarimetric observations of rotating stars based on regularized image-reconstruction procedures implemented in INVERs 10 developed by Piskunov \& Kochukhov (2002), it became possible to invert simultaneously the information of the rotationally modulated Stokes profiles into elemental abundance as well as the three vector components of the stellar magnetic field. In their Papers I and II, Piskunov \& Kochukhov (2002) and Kochukhov \& Piskunov (2002) describe in detail the concept of MDI, the basic numerical techniques to efficiently solve the magnetic radiative transfer, the surface integration of the resulting Stokes profiles and the application of the regularization concept. Applications of INVERS10 are presented in Kochukhov et al. (2002, 2004a) and Folsom et al. (2008).

In case of HD 24712 we only have access to two Stokes parameters $(I$ and $V)$. For this reason we had to resort to multipolar regularization to obtain a solution free from undesirable cross-talks between different field components. This is a reasonable prior model of the Ap-star magnetic field geometry. Numerous previous studies (e.g. Landstreet \& Mathys 2000; Bagnulo et al. 2002) showed that rotational modulation of the integral quantities derived from the circular polarization spectra of Ap stars are well-explained by dipolar or quadrupolar-like fields with some additional influence of abundance inhomogeneities. Multipolar regularization (see
Piskunov \& Kochukhov 2002) has been successfully used for the mapping of the Ap stars $\alpha^{2} \mathrm{CVn}$ (Kochukhov et al. 2002) and HD 72106 (Folsom et al. 2008). This method introduces external constraints similar to the multipolar field parameterization proposed by Bagnulo et al. (1999) and leads INVERS10 to solutions close to a general second-order multipolar expansion. Numerical experiments (Kochukhov \& Piskunov 2002) demonstrated that this technique provides reliable imaging of stars with quasi-dipolar or quadrupolar magnetic fields. However, our previous experience with the MDI shows that using multipolar regularization may prevent us from reconstructing the fine structure of magnetic field by forcing it closer to a low-order multipolar geometry. The alternative, Tikhonov regularization, which does not introduce assumptions about the global geometry of the stellar surface structures, was only applied for reconstruction of chemical abundance. The values of two regularization parameters were adjusted to ensure that at the convergence the total contribution of the regularization terms was roughly $20-80 \%$ of the sum of weighted squared differences between observations and synthetic spectra. We have verified that a variation of regularization parameters by factors of 2-5 leaves little imprint on the final solution.

The additional information content of the Stokes profiles compared to the data used for conventional Doppler Imaging makes it possible to also map stars with rotational velocities as low as that of HD 24712 with $v_{\mathrm{e}} \sin i=5.6 \mathrm{~km} \mathrm{~s}^{-1}$ (derived by Ryabchikova et al. 1997). Nevertheless we would like to state here that due to this very low $v_{\mathrm{e}} \sin i$, the uncertainties of the latitudes of the abundance enhancement and depletion regions are larger than for stars with $v_{\mathrm{e}} \sin i$ higher than $\simeq 10 \mathrm{~km} \mathrm{~s}^{-1}$. On the other hand, the longitude position of the surface features is mainly constrained by the phase coverage, which is fairly good for our observations. Information contained in the considerable rotational modulation of the line intensities due to changing visibility of abundance spots on the surface of HD 24712 allows us to infer the longitudinal position of large-scale abundance structures with sufficient precision. Numerical experiments demonstrate that longitudinal shifts of major abundance features by $\sim 10^{\circ}$ (few 0.01 in phase units) can be readily detected with our observational data.

\subsection{Magnetic field geometry}

Determining the geometry of the magnetic field on the surface of HD 24712 was performed choosing seven different Fe I and five different Nd III lines, which are listed in Table 3. These lines were chosen because their Landé factors and Zeeman splitting patterns yield strong polarization signatures, they are not affected by severe blending, and they show clear variations in the strength of the Stokes I profiles due to abundance inhomogeneities and, to a lesser extent, due to variable Zeeman effect. Furthermore, the lines of $\mathrm{Fe}$ and $\mathrm{Nd}$ exhibit an opposite variation with rotation phase. Thus, using these two elements simultaneously in a self-consistent abundance and magnetic inversion yields a more robust reconstruction of the stellar magnetic field topology.

Important parameters needed as input for the MDI procedure are the inclination and the azimuth angle of the stellar rotational axis, $i$ and $\Theta$. It should be mentioned that when mapping with Stokes $I$ and $V$ components, the azimuth angle is irrelevant and we cannot distinguish between $i$ and $180^{\circ}-i$. However, we adopted $i>90^{\circ}$ to be consistent with the analysis of Bagnulo et al. (1995), who determined both angles based on the traditional circular- and on broadband linear polarization 

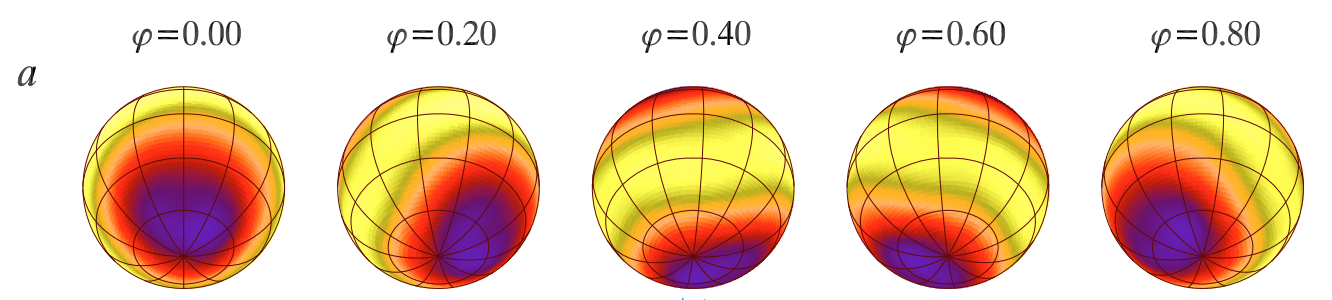

$\mathrm{B}(\mathrm{kG})$

$b$
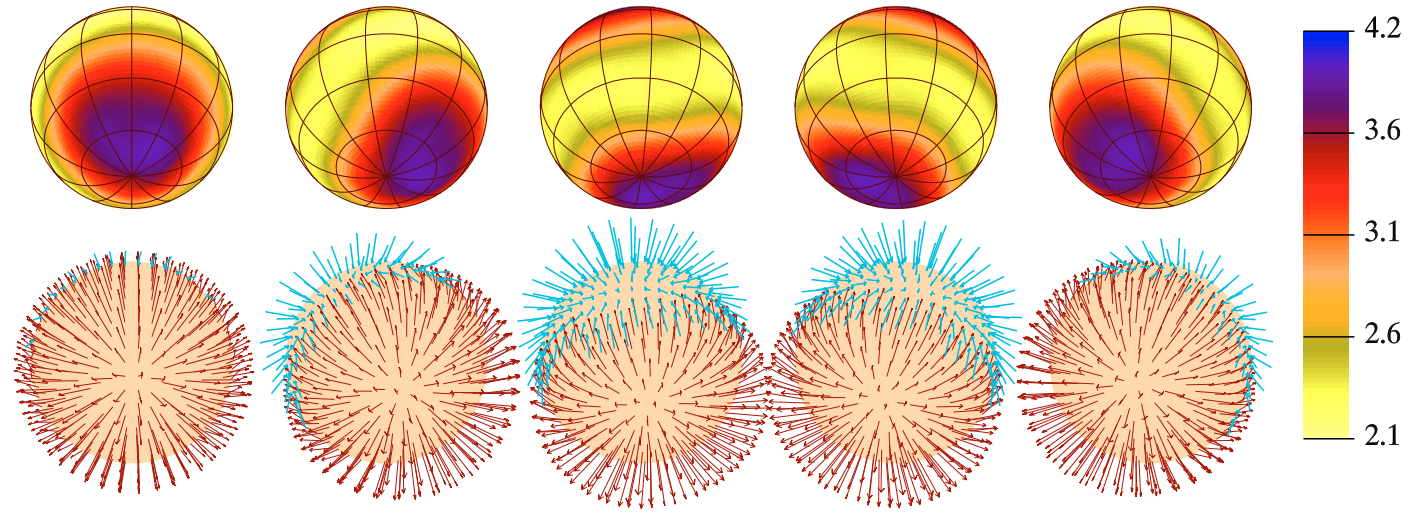

Fig. 1. Mapping of the distribution of magnetic field strength a) and field orientation b) on the surface of the roAp star HD 24712, as derived with modelling the Stokes $I$ and $V$ parameter stellar observations. The results reveal a dominant dipolar magnetic field geometry on the surface of this star with the field strength varying between $2.1 \mathrm{kG}$ and $4.2 \mathrm{kG}$.
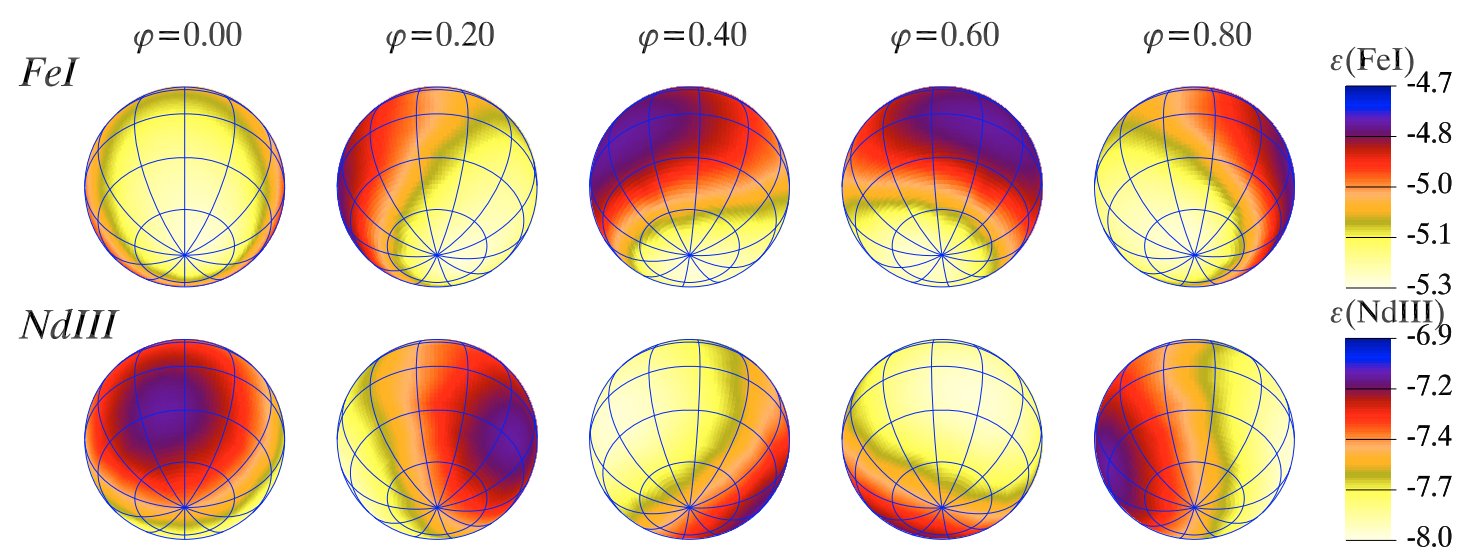

Fig. 2. The abundance distributions of Fe and Nd on the surface of HD 24712. The upper panel shows the surface map of the Fe abundance and the lower panel presents the distribution of Nd derived from Nd III lines. These maps were derived using the Stokes $I$ and $V$ parameter spectra as presented in Fig. 3. In this and other similar plots we use the $\varepsilon(X i)$ to denote abundance, in the $\log \left(N_{\mathrm{X}} / N_{\mathrm{tot}}\right)$ units of the element " $X$ ", derived from lines belonging to the ionization stage " $i$ ".

observations and found $i=137^{\circ}$ and $\Theta=4^{\circ}$. Adopting these values, a surface magnetic map very close to a dipolar geometry yielded the best fit to the observed line profiles. The magnetic field strength varies between $2.1 \mathrm{kG}$ and $4.2 \mathrm{kG}$. In Figs. 1 and 3 the observed line profiles of the chosen Fe I and Nd III lines (dots), the corresponding fit reached during the inversions (lines) and the resulting magnetic field geometry are presented. The near dipolar structure agrees very well with the dipolar model proposed by Bagnulo et al. (1995), only differing in the polar field strength, where they obtain $B_{\mathrm{p}}=3.9 \mathrm{kG}$, compared to $4.2 \mathrm{kG}$ derived from magnetic Doppler imaging.

\subsection{Fe and Nd abundance distribution}

The surface abundances of Fe and Nd mapped simultaneously with the stellar magnetic field geometry are shown in Fig. 2. As mentioned above, these distributions were calculated using seven Fe I and five Nd III lines listed in Table 3. One can easily see that abundances of both elements are quite globally structured. Fe varies between -5.3 and $-4.7 \mathrm{dex}$ in the $\log \left(N / N_{\text {tot }}\right)$, in its maximum, still slightly underabundant compared to the solar value $(-4.59 \mathrm{dex})$. $\mathrm{Nd}$ abundance being derived from $\mathrm{Nd}$ III lines is very high and it varies between -8.0 and -6.9 dex (compared to -10.59 dex solar value). As we see further in Sect. 4.3.3 Nd abundance distribution from Nd II lines is similar in shape but by 1.5 dex smaller in magnitude following the known REE anomaly observed in all roAp stars (Ryabchikova et al. 2001, 2004). Fe and Nd seem to be perfectly anticorrelated: $\mathrm{Fe}$ is accumulated where $\mathrm{Nd}$ is depleted, and minimum $\mathrm{Fe}$ abundance can be found where $\mathrm{Nd}$ is at its maximum.

Comparing the patterns of these two elements to the derived magnetic field geometry presented in Fig. 1, we find that the Fe abundance enhancement region is associated with the area where the magnetic equator dominates the visible stellar hemisphere, around $\phi \simeq 0.5$, whereas the Nd map exhibits its area of maximum abundance around the phase where the positive magnetic pole is oriented towards the observer. Due to the orientation of the star we are not able to directly observe the negative magnetic pole.

It is surprising to see that, contrary to our expectations, both elements are not accumulated or depleted on both magnetic poles or around the magnetic equator. Furthermore, the center of maximum or minimum abundance, respectively, seems to be shifted in latitude and/or longitude with respect to the magnetic polar regions, not only for $\mathrm{Nd}$, but also for several other elements presented in Sect. 4.3. 
T. Lüftinger et al.: First magnetic Doppler images of an roAp star

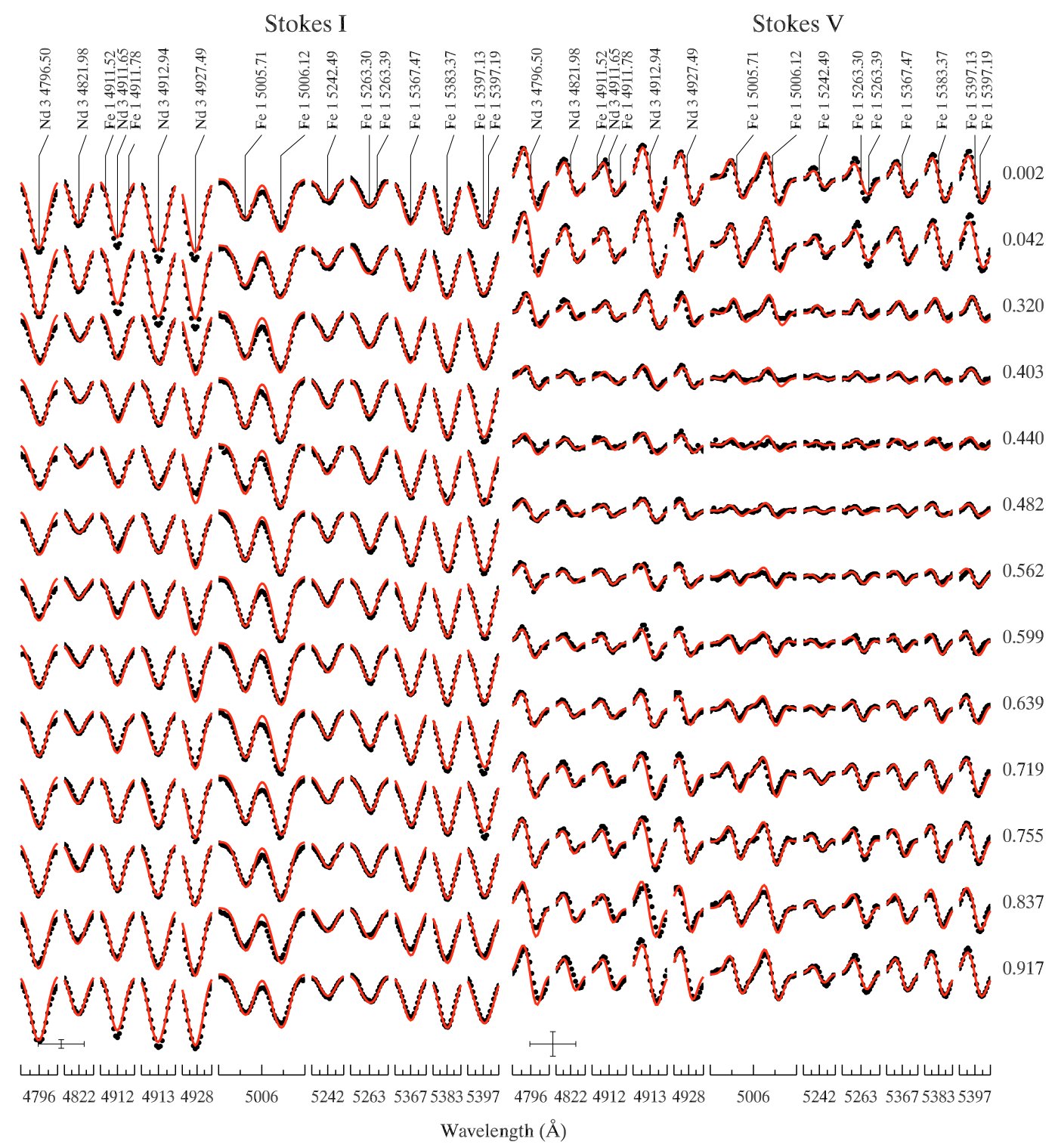

Fig. 3. Comparison of the observed (dots) and calculated (lines) Stokes $I$ and $V$ profiles. Spectra are shown with increasing phase value (listed in Table 2) from top to bottom. The bars at the lower left and middle show the vertical (5\%) and horizontal (0.5 $\AA$ ) scale.

\subsection{Surface abundances of individual elements}

After having derived the magnetic field geometry of HD 24712 using the Fe I and Nd III lines, we were able to recover abundances of sixteen additional ions applying the INVERS10 code and the previously determined fixed magnetic map.

\subsubsection{Magnesium, calcium, and scandium}

The surface abundance distribution of magnesium was modelled using one $\mathrm{Mg}$ I line at $5528 \AA$ with INVERS 10 , and we found a remarkable variation between -6.0 and -4.5 dex. $\mathrm{Mg}$ is, as Sc, $\mathrm{Ti}, \mathrm{Fe}$, and $\mathrm{Ni}$ depleted (underabundant by up to $1.5 \mathrm{dex}$ compared to the solar value) at the phase where the positive magnetic pole dominates the visible stellar surface and shows a single, huge overabundance region around the magnetic equatorial zone, indicating that the center of maximum abundance does not coincide with the (suspected) negative magnetic pole.

For calcium as well as for chromium (see Sect. 4.3.2) we found only a marginal abundance variation not exceeding
0.2 dex and consequently do not consider our mapping results as significant.

Scandium exhibits a variation of 1.2 dex over the stellar surface, between -8.9 and -10.1 dex, also accumulated where the magnetic equatorial region dominates the visible stellar surface, whereby the central region of the depleted area occurs at similar longitude as that of $\mathrm{Mg}$ and $\mathrm{Fe}$ and the central region of maximum abundance precedes that of $\mathrm{Mg}$ and $\mathrm{Fe}$ by about $60^{\circ}$. The region of enhanced $S c$ abundance seems to occupy a larger fraction on the surface of HD 24712 than numerous other elements. At its maximum abundance phase, the $S c$ value corresponds to the solar one of 8.99 dex.

\subsubsection{Iron-peak elements}

Two lines of ionized titanium were used to recover the surface abundance structure of this element (Fig. 4). As Mg and Sc, it shows a tendency to accumulate near the magnetic equatorial region, exhibiting near solar concentration, and the abundance decreases to -7.8 dex where the positive magnetic pole is visible. 


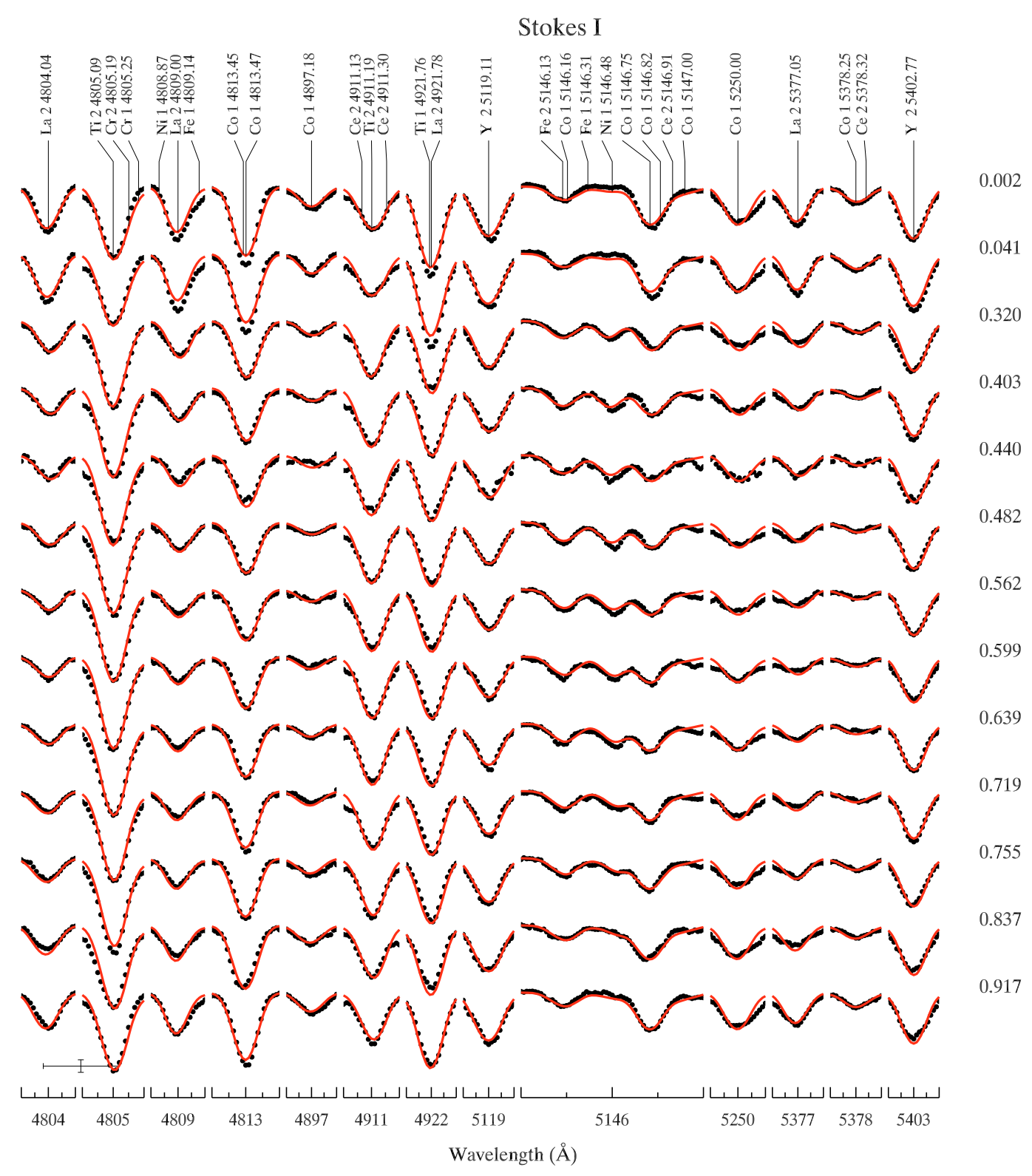

Fig. 4. Comparison of the observed (dots) and calculated (lines) Stokes $I$ and $V$ profiles used for mapping of Ti, Co, Y and La. Spectra are plot with increasing phase value (listed in Table 2) from top to bottom. The bars at the lower left and middle again show the vertical (5\%) and horizontal $(0.5 \AA)$ scale. Note the total disappearance and reappearance of the Ni I line at $5146 \AA$.

For chromium only one line around $5510 \AA$ could be used as input for INVERS10 and we obtained just a marginal (insignificant as for calcium, see Sect. 4.3.2) variation between -5.9 and -6.1 dex.

Cobalt, as Y and all the REE, is clearly accumulated on the surface region visible at the phase where the positive magnetic pole dominates, and less abundant around the magnetic equatorial region, varying between -5.8 to $-6.4 \mathrm{dex}$. Co is significantly overabundant with respect to the Sun $(-7.12$ dex $)$ over the whole stellar surface. The element was mapped taking into account six Co I lines shown in Fig. 4 and listed in Table 3.

A very interesting example of enormous line profile variation has been discovered for the nickel lines, Ni I at $5035 \AA$, and $5146 \AA$. The surface map derived from the single unblended line at $5035 \AA$ reveals a huge, high contrast region of overabundance at phase 0.5 and a region of underabundance down to $-7.6 \mathrm{dex}$ at the phase where the positive magnetic pole dominates the visible stellar surface. We noticed that the Ni I $\lambda 5146.48 \AA$ line totally disappears at this rotational phase. In order to check the abundance derived from the single $5035 \AA$ line and to be able to use the blend of $\mathrm{Ni}$ with $\mathrm{Fe}, \mathrm{Co}$, and $\mathrm{Ce}$ around $5146 \AA$, we used the abundance pattern obtained from the Ni I 5035 line to map $\mathrm{Co}$ and $\mathrm{Ce}$ from the blend mentioned above and got a very good agreement of observed and calculated profiles. The fit to the line profiles reached from this inversion is presented in Fig. 4. Ni distribution, in longitude and in latitude, is very similar to that of $\mathrm{Fe}$, and abundance varies from -7.6 to $-6.3 \mathrm{dex}$, being significantly underabundant compared to the solar value of -5.81 dex.

\subsubsection{Yttrium and the rare-earth elements}

Yttrium varies from -9.0 to -8.5 dex (overabundant up to $1.3 \mathrm{dex}$ compared to the solar value of -9.83) and exhibits a structure closer to that of the REE, with the center of abundance 
T. Lüftinger et al.: First magnetic Doppler images of an roAp star

Table 3. Table of elements and spectral lines used for mapping.

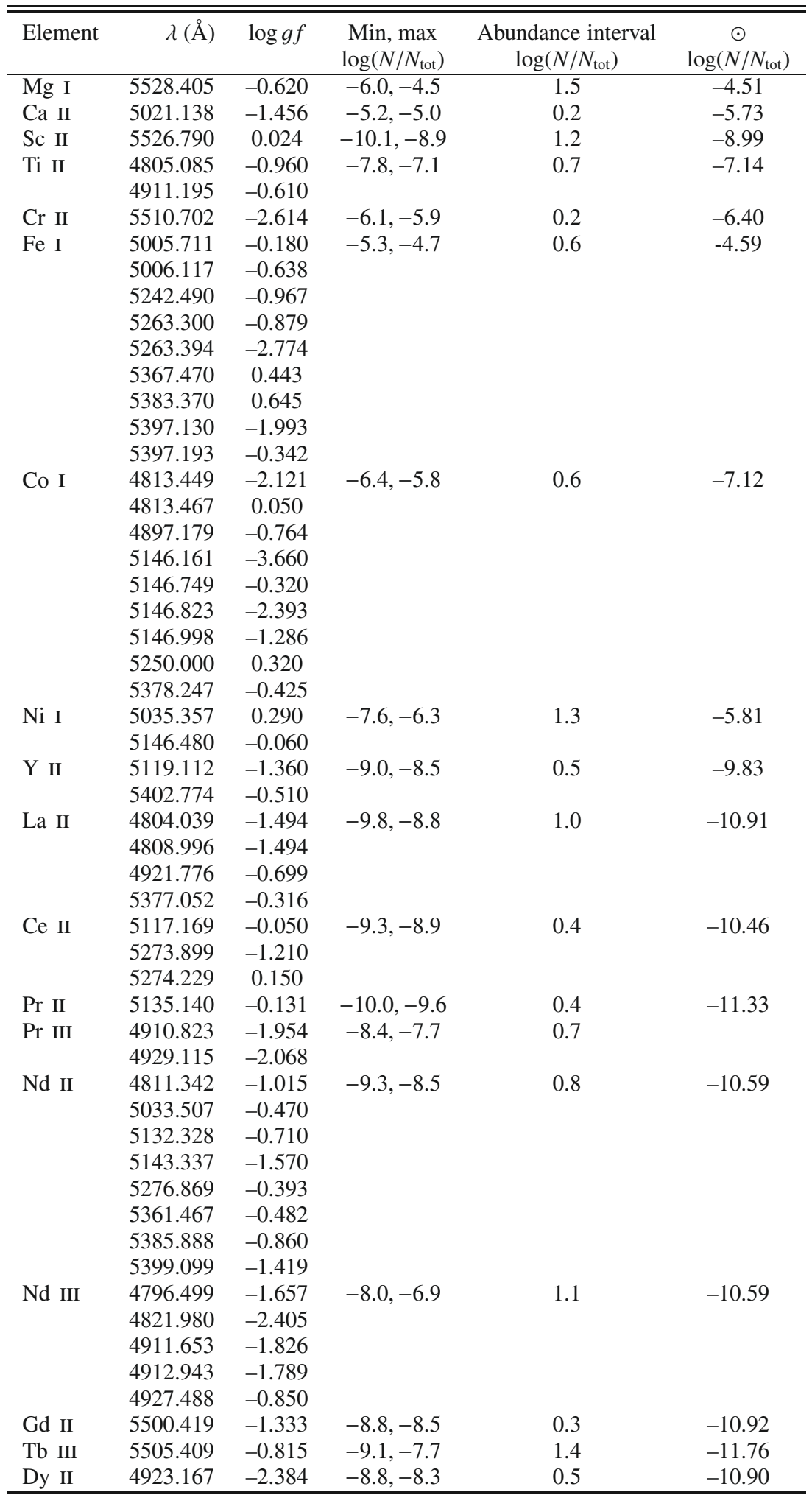

From left to right: element identification, central wavelength, the $\log g f$ values of these lines, abundance interval and the range in dex, and the solar value as a comparison.

maximum shifted to the northernmost part of the visible stellar hemisphere. Yttrium reaches its maximum and minimum, respectively, slightly after that of Nd. As presented in Figs. 6-8, all the REE we mapped are enhanced around the phase where the positive magnetic pole is visible and depleted where the magnetic equatorial region dominates the surface. For REE depletion means the minimum of the observed surface abundance which usually exceeds the solar photospheric abundance. We obtained abundance distributions of $\mathrm{La}, \mathrm{Ce}, \mathrm{Pr}, \mathrm{Nd}, \mathrm{Gd}, \mathrm{Tb}$, and $\mathrm{Dy}$. For Pr and Nd, abundance distributions were calculated from the 


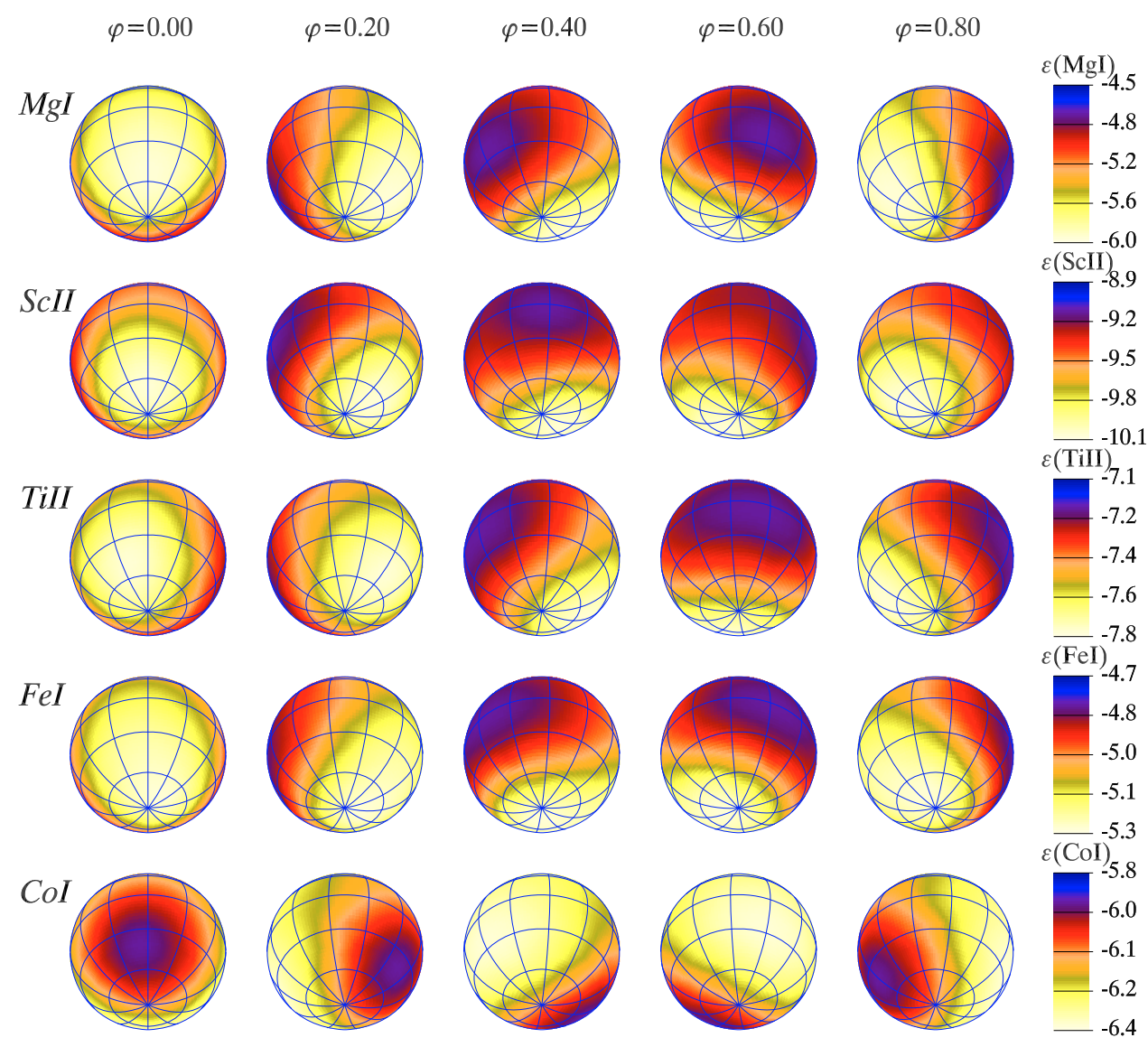

Fig. 5. Abundance distribution of $\mathrm{Mg}, \mathrm{Sc}, \mathrm{Ti}, \mathrm{Fe}$, and Co on the surface of HD 24712 obtained from the lines listed in Table 3.

separate analysis of the lines of the singly and doubly ionized element.

Lanthanum is significantly overabundant compared to the solar value $(-10.91)$ and varies from -9.8 to -8.8 dex. It covers stellar latitudes very similar to those of $\mathrm{Co}, \mathrm{Nd}$, and $\mathrm{Gd}$, although due to the very low $v_{\mathrm{e}} \sin i$ of the star, the latitudes of the abundance enhancement and depletion regions are affected by larger uncertainties than for stars with $v_{\mathrm{e}} \sin i$ higher than $\simeq 10 \mathrm{~km} \mathrm{~s}^{-1}$. The center of maximum abundance slightly lags behind that of $\mathrm{Nd}$.

Variation of Y and La lines is illustrated in Fig. 4.

The cerium surface map was reconstructed using two Ce II lines, also exhibiting the typical overabundance of REE in roAp stars of more than a dex, but varying only moderately over the surface between -9.3 and -8.9 dex.

Two independent maps of praseodymium were derived using the lines of the singly and doubly ionized element. It is caused by the known REE anomaly observed in roAp stars, where average abundance derived from these lines differs by 1.5-2.0 dex (Ryabchikova et al. 2004), and it is not possible therefore to fit line profiles of both ionization stages within the same inversion. Pr II $\lambda 5136 \AA$ and two spectral lines of Pr III around $4910 \AA$ and $4929 \AA$ were used for inversions. Pr II varies moderately between -10.0 and -9.6 dex being derived from one Pr II line, while higher abundances but with the same distribution shape are obtained from Pr III lines. The Pr spot seems to be shifted in latitude relative to the $\mathrm{Nd}$ enhancement region.

Abundances for neodymium were also derived separately from singly and doubly ionized lines due to the large abundance difference between the two ionization stages. The Nd surface map was derived from eight lines of Nd II listed in Table 3 and exhibits the same distribution as that obtained from Nd III lines (see Sect. 4.2). Nd abundance varies from -9.3 to -8.5 dex. As for the Pr case, the abundance difference derived from $\mathrm{Nd}$ II and Nd III lines reaches 1.5 dex.

Gadolinium is concentrated in a spot very similar to that of $\mathrm{Nd}$, close to the positive magnetic pole region, varying moderately between -8.5 and -8.8 dex, and is overabundant nearly up to 2.5 dex relative to the solar value of -10.92 dex.

The doubly ionized terbium line at $5505 \AA$ was used to map the surface distribution of this element. Tb shows a strong overabundance of up to -7.7 dex (compared to the solar value of -11.76 dex) on the surface part appearing at the phases where the positive magnetic pole dominates the visible stellar hemisphere. It is modulated between -9.1 and -7.7 dex, with the center of maximum abundance shifted by $\simeq 30^{\circ}$ to higher phases compared to Co, $\mathrm{Nd}$, and Dy.

Dysprosium can also be found in a spot near the positive magnetic pole with the center of abundance slightly preceding that of e.g. Gd. The element is varying between -8.3 and $-8.8 \mathrm{dex}$ and found to be overabundant by more than $2.0 \mathrm{dex}$ relative to the solar value of -10.90 dex.

\section{Discussion}

Observing line profiles in several polarization states of stellar radiation and modelling their shapes with magnetic Doppler imaging, we could derive the magnetic field geometry and abundance distributions of numerous elements inhomogeneously distributed over the surface of the roAp star HD 24712. This is the 


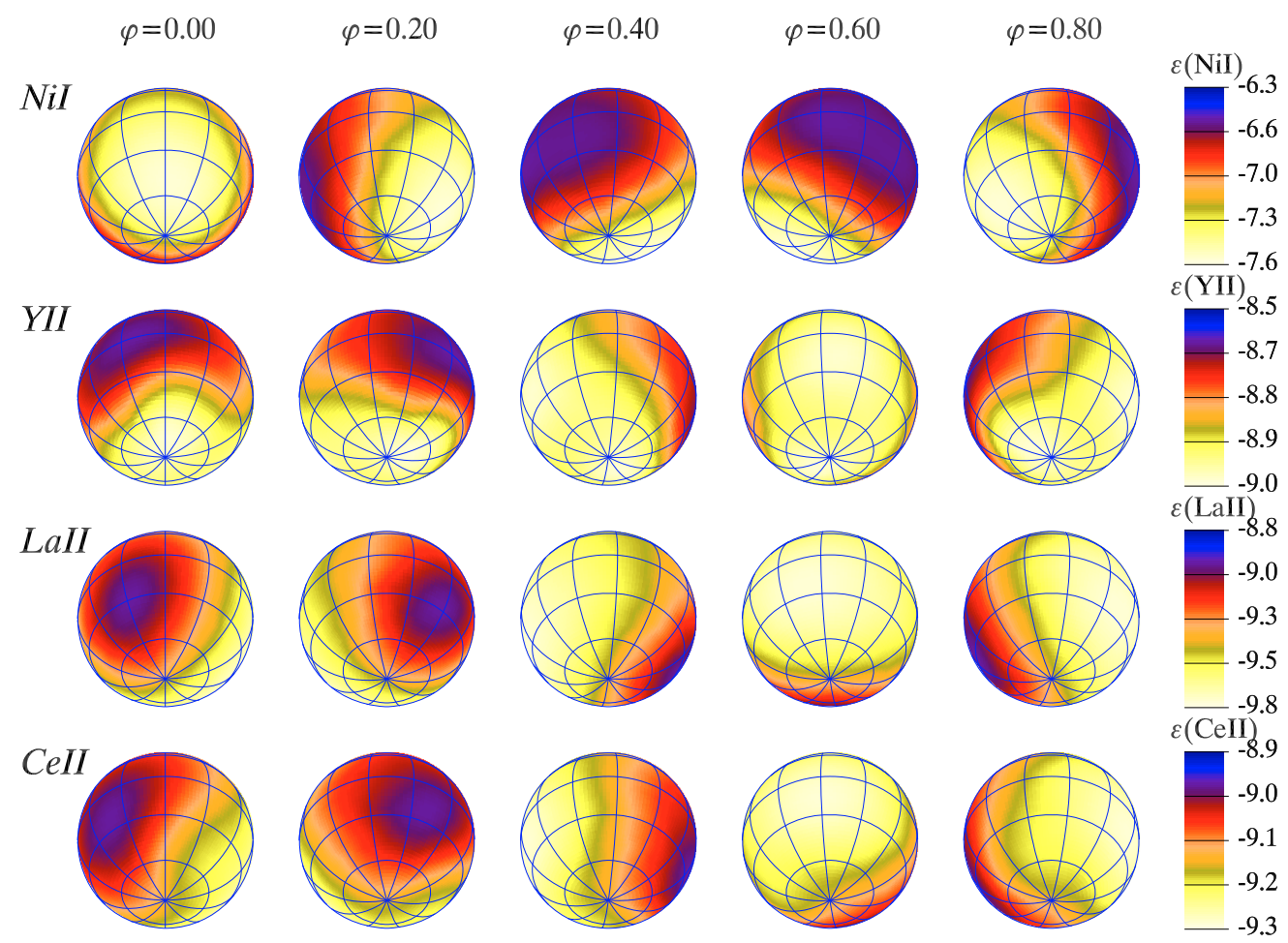

Fig. 6. Surface abundance distribution of Ni, Y, La, and Ce (rom top to bottom) on the surface of HD 24712. The maps were obtained taking into account the lines listed in Table 3.

first analysis of this kind for a magnetic pulsating star. Our investigation shows a complex picture of the interplay between magnetic field, pulsation and atmospheric inhomogeneities in this prototypical roAp star. However, the low $v_{\mathrm{e}} \sin i$ of the star and the limited spectral resolution of our spectropolarimetric observations did not allow us to reliably recover all but the largest spatial scales of the abundance and magnetic structures. For this reason we will discuss only the gross properties of the chemical spots and the magnetic field, without touching upon the higherorder moments of the surface structures.

We obtain a dominant dipolar magnetic field structure (which might be more complex, though the general picture is certainly dipolar) and elemental abundance patterns that are correlated to this geometry in an unexpected way: instead of abundance enhancement regions on both magnetic poles or around the magnetic equator, we observe huge enhancement or depletion regions around either the phase where the positive magnetic pole is visible or where the magnetic equatorial region dominates the visible stellar surface.

In addition, even more exciting and novel is the fact that these enhancement or depletion regions for various chemical elements are shifted in longitude relative to each other. The center of $\mathrm{Mg}$ underabundance as well as that of $\mathrm{Sc}, \mathrm{Fe}$, and $\mathrm{Ni}$ can be found at phase 0.0 , while that of Ti occurs around phase 0.082 . The center of maximum abundance of $\mathrm{Tb}$ appears very close to phase $\varphi=0.0$, that of Co and Gd slightly after $\varphi=0.0$, around $0.02, \mathrm{Nd}$ spots appear at $\varphi=0.04$, Pr and Dy at around $\varphi=0.06$. Y and La are already shifted by $30^{\circ}$ or $\approx 0.082$ in the direction of increasing phase value. Ce, whose center of maximum abundance lags behind all other elements, is shifted by as much as $\approx 0.12$ or $\approx 45^{\circ}$.

To investigate the reliability of these longitudinal offsets of the abundance structures we have performed a forward calculation for Y, La, and Ce showing most of the conspicuous shifts.
Using the final abundance maps reconstructed for these elements we have synthesized their line profiles, rotating surface structures in the longitudinal direction by small amounts and compared the resulting modification of Stokes $I$ with the typical observational uncertainty. This comparison is illustrated in Fig. 9. As is evident from this numerical experiment, the high $S / N$ of our observations enables us to easily detect shifts of $\gtrsim 0.05$ for $\mathrm{Y}$ and $\mathrm{Ce}$ and even smaller shifts for La. Thus the relative longitudinal positions of spots of different elements are precise to within $0.02-0.05$ of the rotation period.

The diverse surface abundance behaviour observed in HD 24712 most likely gives us information on how the magnetic field influences the chemical diffusion of the various species within the stellar atmosphere. Accordingly we tried to correlate our results with simple atomic parameters (e.g., atomic weight or excitation potential), but did not find any clear evidence for such a connection. This gives rise to the speculation, as the effect is clearly present, that there is a more complex correlation of the various atomic characteristics influencing chemical diffusion through stellar atmospheres in presence of a magnetic field. Thus these relative shifts in longitude of the mapped elements provide valuable constraints for diffusion models that try to explain how the magnetic field and other hydrodynamical processes regulate atmospheric diffusion.

Moreover, we obtain shifts in latitude of abundance enhancement regions exhibiting otherwise comparable accumulation tendencies. Sc, Ti, Fe, and $\mathrm{Ni}$ in this context appear in similar latitudes, whereas $\mathrm{Mg}$ and Co seem to be shifted to lower latitudes by $30^{\circ}$ and $45^{\circ}$, respectively, compared to the above mentioned elements.

Among the elements belonging to the group that is enhanced around the positive magnetic pole, $\mathrm{Tb}$ and $\mathrm{Y}$ seem to cover the highest latitudes, $\mathrm{Ce}$ and $\mathrm{Pr}$ are found to be shifted to lower 


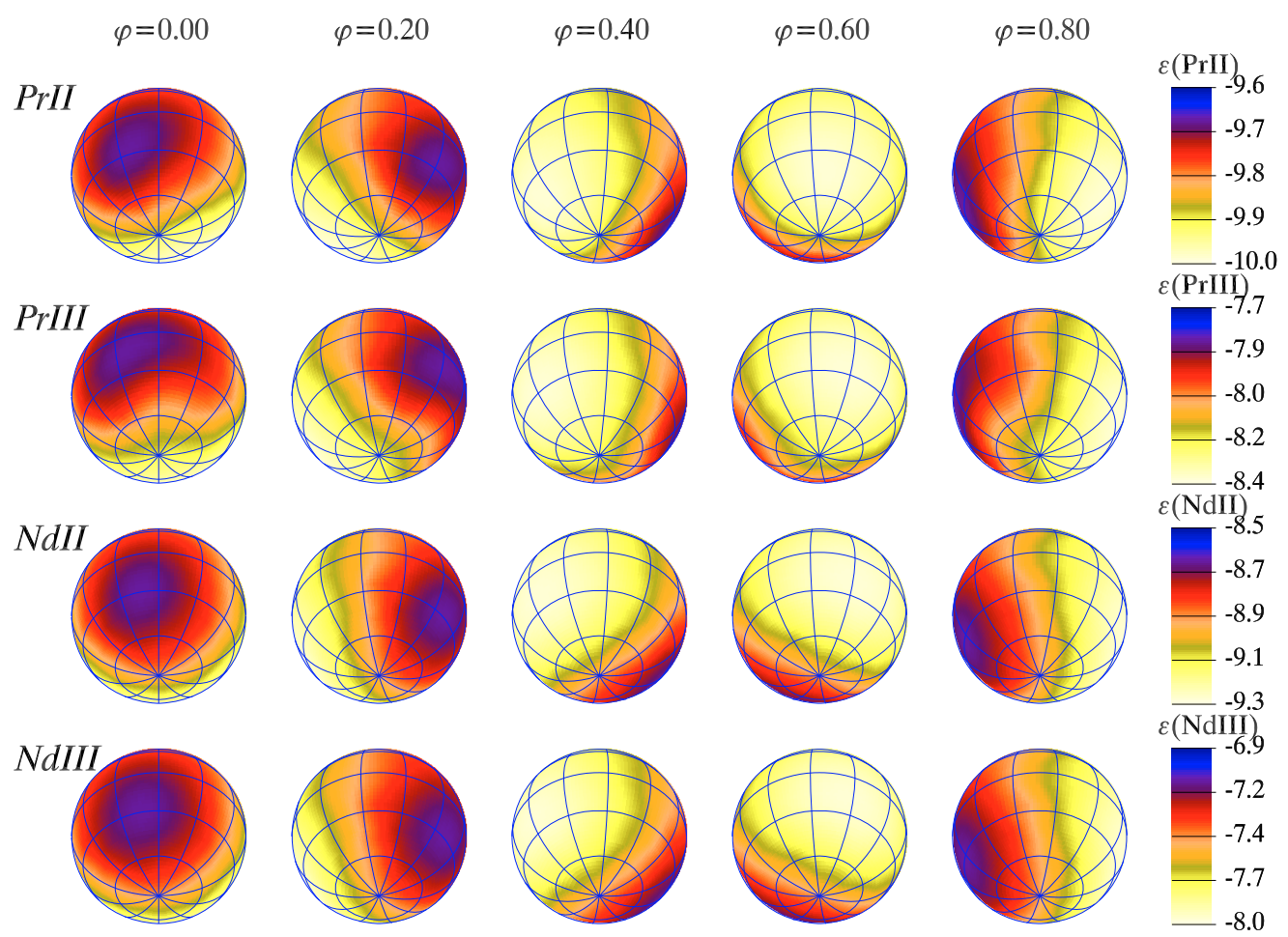

Fig. 7. Elemental abundance distribution on the surface of HD 24712. Maps of Pr and Nd derived separately from the lines of the first and second ionization stages of both elements are presented.

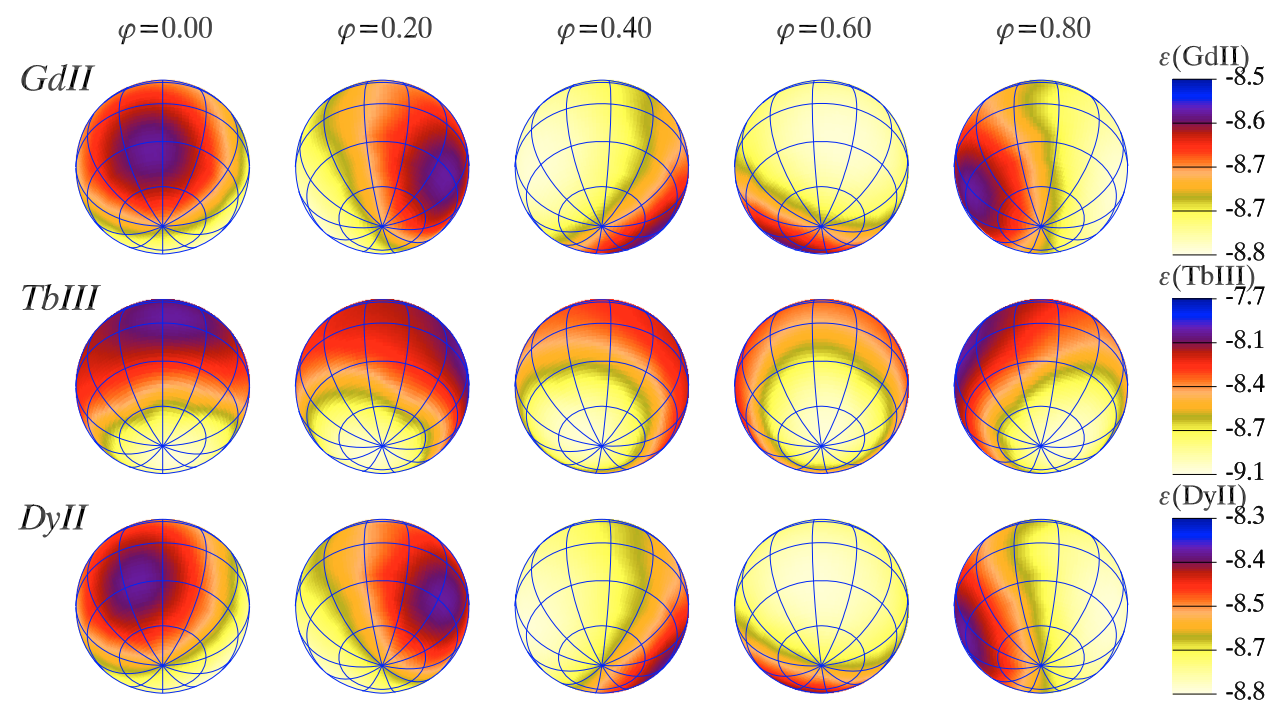

Fig. 8. Surface abundance distributions of $\mathrm{Gd}, \mathrm{Tb}$, and Dy derived from the lines listed in Table 3.

latitudes by around $15^{\circ}$, and $\mathrm{La}, \mathrm{Nd}, \mathrm{Gd}$, and Dy spots are shifted even more southwards by $\approx 30^{\circ}$.

This finding helps to explain the phase shift between pulsation curves of spectral lines belonging to different REE elements, for instance, $\mathrm{Pr}$ and $\mathrm{Nd}$, that we found in the recent study by Ryabchikova et al. (2007). According to the NLTE analysis of $\mathrm{Nd}$ II, III and Pr II, III line formation in the atmosphere of HD 24712 (Mashonkina et al. 2005, 2009), the lines of both elements are formed at nearly the same atmospheric layers. However, the pulsation analysis of Ryabchikova et al. (2007) shows that they are shifted by 0.1 in the pulsation phase relative to each other. According to the theoretical calculations by Sousa \& Cunha (2008) and numerical simulations by Khomenko \& Kochukhov (2009), the amplitude and phase distributions with height in roAp atmospheres should depend on the local magnetic field strength and inclination. Slightly different location of $\mathrm{Pr}$ and $\mathrm{Nd}$ spots relative to the magnetic field pole may therefore be a reason for the pulsation amplitude and phase difference observed in Pr and Nd lines. This illustrates the potential and importance of MDI when interpreting pulsational observations of roAp stars.

Vertically stratified abundances are very likely present in the atmosphere of HD 24712 (e.g., Ryabchikova et al. 2008). In particular, a major discrepancy between two ionization states of the 
La

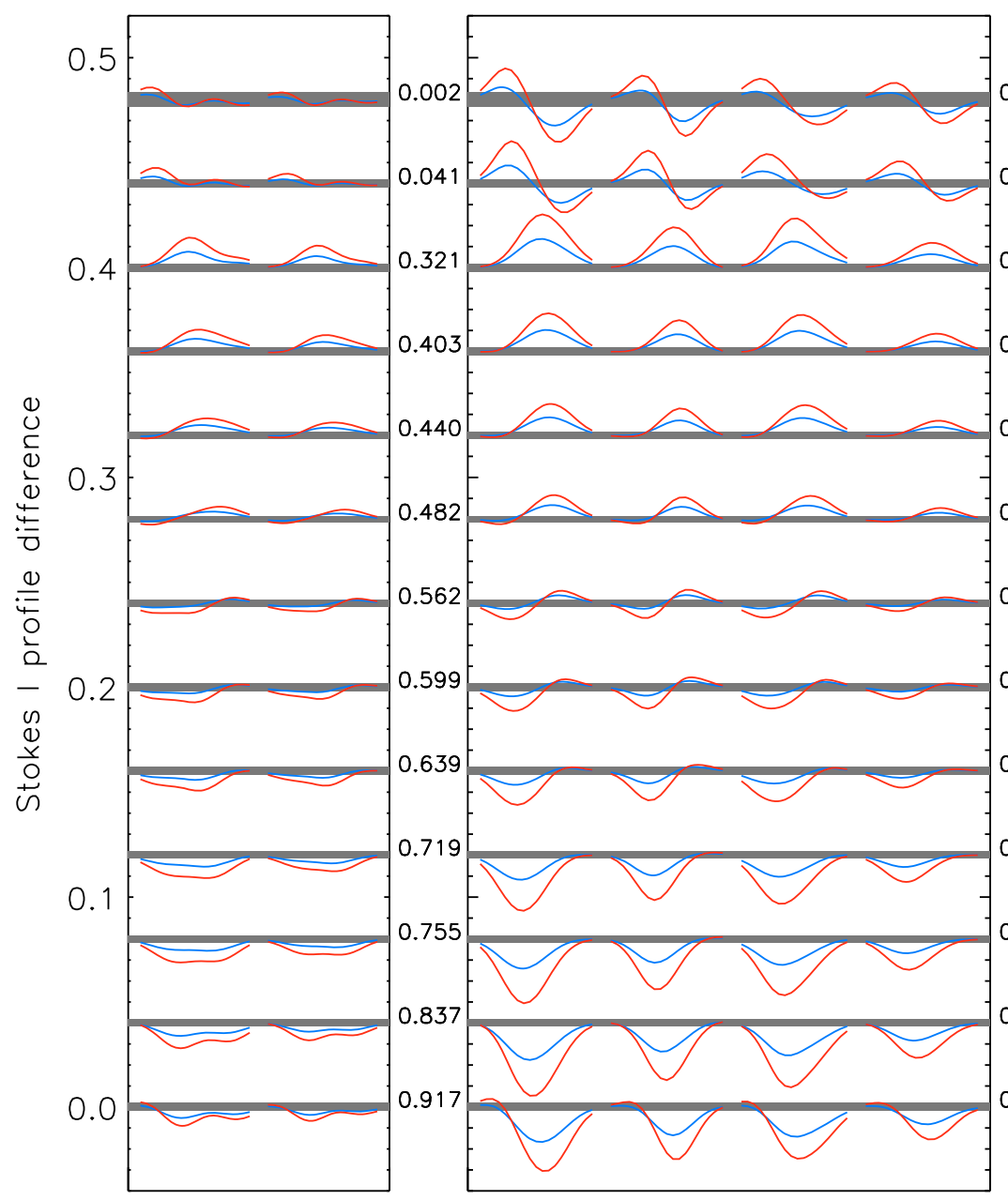

$\mathrm{Ce}$

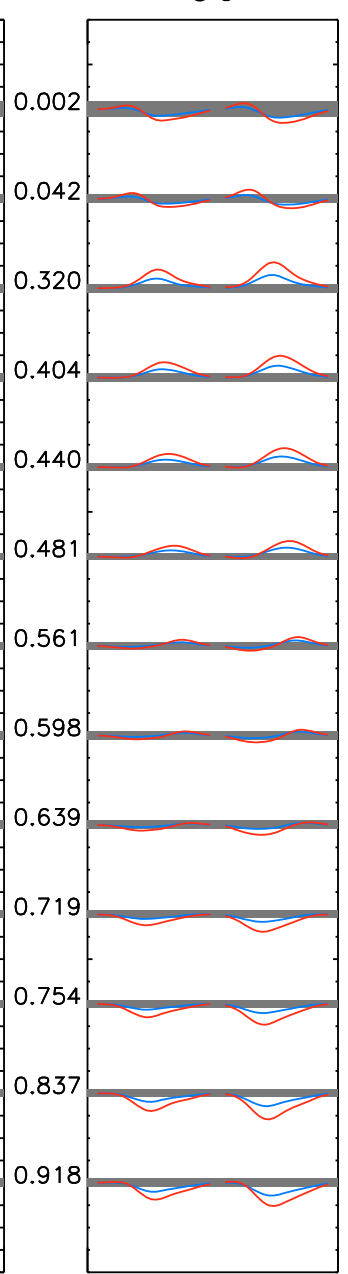

Fig. 9. Effect of a small rotation of the surface structure of Y, La, and Ce on the Stokes I profiles of the lines of these elements used for mapping. Panels show the difference between the original synthetic profiles and spectra computed with the surface spots shifted by 0.05 (blue) and 0.10 (red) of the rotation period $\left(18^{\circ}\right.$ and $36^{\circ}$ on the stellar surface, respectively). The grey bar indicates the uncertainty $( \pm \sigma)$ of the spectra corresponding to the $S / N$ of individual rotation phases. The difference spectra are offset vertically for display purposes. The horizontal scale is arbitrary.

same REE element is attributed to stratification (Mashonkina et al. 2009). To estimate the impact of stratification, we performed an analysis of the vertical gradient of $\mathrm{Fe}$ abundance (Lüftinger et al. 2004) during the phase where the magnetic equatorial region dominates the stellar hemisphere and also where the positive magnetic pole is visible, choosing Fe lines that are most sensitive to stratification. Fe turned out to be significantly stratified, with abundance changing by $\approx 2$ dex between the upper and lower parts of the atmosphere of HD 24712. We also found a slight difference in the location of the abundance jump comparing results from the two extreme phases. In order to keep MDI results influenced as little as possible by the stratification effects, we chose lines for mapping that are hardly affected by a changing vertical distribution. It is still possible however that part of the horizontal abundance structure we find is due to variation of the chemical stratification profile across the stellar surface.

Currently, there are no theoretical diffusion models that can provide quantitative explanation of the abundance maps derived in our Doppler imaging study of HD 24712. For this reason we are not able to compare the results of our investigation to extensive theoretical predictions for individual elements in the atmospheres of Ap stars. The standard diffusion calculations consider chemical transport processes in the presence of axisymmetric magnetic field and thus anticipate abundance inhomogeneities to be axially symmetric themselves (Michaud et al. 1981). These predictions of the simple diffusion theory are inconsistent with the MDI results obtained for HD 24712. We find a nearly dipolar magnetic field geometry coexisting with diverse abundance distributions, frequently shifted relative to the magnetic field axis. A similar inconsistency between a simple magnetic field topology and complex abundance distributions was found in other recent DI studies of Ap stars (Kochukhov et al. 2002, 2004b). It is possible that diffusion processes are sensitive to the small scale magnetic structures that could be resolved only with the full Stokes vector MDI, such as the study carried out by Kochukhov et al. (2004a) for 53 Cam. On the other hand one can also suspect that the current simple diffusion theory lacks certain physical processes other than the magnetic field, that can influence or even drive chemical spot formation. Recent discovery of the evolving chemical clouds in the atmosphere of non-magnetic chemically peculiar stars (Kochukhov et al. 2007) has demonstrated that the surface structure formation in the upper main sequence stars is not necessarily driven by the magnetic field alone.

Anticipating further development of theoretical aspects, we provide new information for the diffusion properties within the atmosphere of a cool Ap star, obtain new insights in the 
atmospheric structure and the field geometry, the origin and interplay of abundance variations, pulsation and magnetic fields in these unique stellar laboratories.

Acknowledgements. This work was supported by the Austrian Science Fund (FWF-PP17890) and by grant 11630102 from the Royal Swedish Academy of Sciences. T.R. acknowledges financial support from RFBR grant 08-02-00469a and from the RAS Presidium (Program "Origin and Evolution of Stars and Galaxies"). O.K. is a Royal Swedish Academy of Sciences Research Fellow supported by a grant from the Knut and Alice Wallenberg Foundation.

\section{References}

Bagnulo, S., Landolfi, M., \& Degl'Innocenti, M. L. 1999, A\&A, 343, 865 Babel, J. 1992, A\&A, 258, 449

Bagnulo, S., Landi Degl'Innocenti, E., Landolfi, M., \& Leroy, J. L. 1995, A\&A, 295, 459

Bagnulo, S., Landi Degl'Innocenti, M., Landolfi, M., \& Mathys, G. 2002, A\&A, 394, 1023

Cunha, M. S. 2006, MNRAS, 365, 153

Cunha, M. S., Fernandes J. M. M. B., \& Monteiro, M. J. P. F. G. 2003, MNRAS, 343,831

Donati, J.-F., \& Collier Cameron, A. 1997, MNRAS, 291, 1

Donati, J.-F., Howarth, I. D., Jardine, M. M., et al. 2006, MNRAS, 370, 629

Folsom, C. P., Wade, G. A., Kochukhov, O., et al. 2008, MNRAS, 391, 901

Glagolevskii, Y. V., Piskunov, N. E., \& Khokhlova, V. L. 1985, SvAL, 11, 154

Hussain, G. A. J., Jardine, M., \& Collier Cameron, A. 2001, MNRAS, 322, 681

Ilyin, I. V. 2000, High resolution SOFIN CCD échelle spectroscopy, Ph.D. Thesis, University of Oulu

Khomenko, E., \& Kochukhov, O. 2009, in Cosmic Magnetic Fields: From Planets, to Stars and Galaxies, IAU Symp., 259, 409

Kochukhov, O., \& Piskunov, N. 2002, A\&A, 388, 868

Kochukhov, O., Piskunov, N., Ilyin, I., et al. 2002, A\&A, 389, 420

Kochukhov, O., Bagnulo, S., Wade, G. A., et al. 2004a, A\&A, 414, 613

Kochukhov, O., Drake, N. A., Piskunov, N., \& de la Reza, R. 2004b, A\&A, 424, 935

Kochukhov, O., Adelman, S. J., Gulliver, A. F., \& Piskunov, N. 2007, Nature Phys., 3, 526
Kupka, F., Piskunov, N., Ryabchikova, T. A., et al. 1999, A\&AS, 138, 119 Kurtz, D. W. 1982, MNRAS, 200, 807

Kurtz, D. W., \& Martinez, P. 2000, Baltic Astron., 9, 253

Kurtz, D. W., Kawaler, S. D., Riddle, R. L., et al. 2002, MNRAS, 330, L57

Landstreet, J. D., \& Mathys, G. 2000, A\&A, 359, 213

Lüftinger, T., Kochukhov, O., Ryabchikova, T., Ilyin, I., \& Weiss, W. W. 2004, The A-Star Puzzle, held in Poprad, Slovakia, July 8-13, ed. J. Zverko, J. Ziznovsky, S. J. Adelman, \& W. W. Weiss, IAU Symp., 224 (Cambridge, UK: Cambridge University Press), 253

Mashonkina, L., Ryabchikova, T., \& Ryabtsev, A. 2005, A\&A, 441, 309

Mashonkina, L., Ryabchikova, T., Ryabtsev, A., \& Kildiyarova, R. 2009, A\&A, 495, 297

Matthews, J. M., Wehlau, W. H., Walker, G. A. H., \& Yang, S. 1988, ApJ, 324, 1099

Michaud, G. 1970, ApJ, 160, 640

Michaud, G., Charland, Y., \& Megessier, C. 1981, A\&A, 103, 244

Piskunov, N. E. 1985, SvAL, 11, 18

Piskunov, N. E., Kupka, F., Ryabchikova, T. A., et al. 1995, A\&AS, 112, 525

Piskunov, N., \& Kochukhov, O. 2002, A\&A, 381, 736

Ryabchikova, T. A., Landstreet, J. D., Gelbmann, M. J., et al. 1997, A\&A, 327, 1137

Ryabchikova, T. A., Piskunov, N. E., Stempels, H. C., et al. 1999, Phys. Scr., 83, 162

Ryabchikova, T. A., Tsymbal, V. V., Malanushenko, V. P., \& Savanov, I. S. 2000, in Magnetic Fields of Chemically Peculiar and Related Stars, ed. Yu. V. Glagolevskij, \& I. I. Romanyuk, Moscow, 180

Ryabchikova, T. A., Savanov, I. S., Malanushenko, V. P., \& Kudryavtsev, D. O. 2001, Astron. Rep., 45, 382

Ryabchikova, T., Nesvacil, N., Weiss, W. W, Kochukhov, O., \& Stütz, Ch. 2004, A\&A, 423, 705

Ryabchikova, T., Wade, G. A., Auriére, M., et al. 2005, A\&A, 429, L55

Ryabchikova, T., Sachkov, M., Weiss, W. W., et al. 2007, A\&A, 462, 1103

Ryabchikova, T., Kochukhov, O., \& Bagnulo, S. 2008, A\&A, 480, 811

Stibbs, D. W. N. 1950, MNRAS, 110, 410

Sousa J. C., \& Cunha, M. S. 2008, CP\#Ap Workshop, ed. J. Žižňovský, J. Zverko, E. Paunzen, \& M. Netopil, Contrib. Astr. Obs. Skalnaté Pleso, 38, 453

Walker, G., Matthews, J. M., Kuschnig, R., et al. 2003, PASP, 115, 1023

Wolff, S. C., \& Morrison, N. D. 1973, PASP, 85, 141 